\title{
Arquitetura, Design e Visualização da Informação (Dados): as diferentes fases dos dados entre a percepção e o esclarecimento
}

\author{
- José Neto de Faria \\ Karine Itao Palos \\ Universidade Anhembi Morumbi, Brasil \\ Universidade Anhembi Morumbi, Brasil \\ josenetodesigner@yahoo.com.br \\ karineipa@hotmail.com \\ - Mirtes Marins Oliveira \\ Universidade Anhembi Morumbi \\ mirtescmoliveira@gmail.com
}

Architecture, Design and Information Visualization (Data): different phases of data between the perception and clarification

\begin{abstract}
The article develops a reflection on the different stages of information (data) in the relations of mediation between 'individual interpreter' and 'information systems', and the agency of the processes of perception, interpretation and clarification. The goal was to identify, understand and describe how 'information systems' enact the different stages of articulation of 'information', from "propensity" to 'concatenation', and from 'critical' to the 'action'. Tests and analysis of the effects of 'information systems' were observed in 'narrative accounts' of 'individual interpreter'. However, the conclusion is that the social conformation of 'strategies for obtaining information' prevails on the properties of 'information system'.
\end{abstract}

Keywords: Design, Information, Information Architecture, Information Design, Information Visualization

\section{Introdução}

A visão historicizada, circunscrita por determinados contextos históricos, do desenvolvimento da 'Arquitetura da Informação', do 'Design da Informação' e da 'Visualização da Informação', possui o mérito de descrever o estado técnico, dos métodos de representação, de processamento de espacialização e de visualização da 'informação' (dados), mas como catálogo de boas práticas sedimentadas através do tempo, parece carecer de habilidades capazes de induzir a realização de pesquisas que foquem na compreensão do modo como funcionam os distintos 'sistemas de informação' (objetos técnicos), vistos como depositórios passivos e ativos de 'sentidos imanentes'. As regularidades, presentes nas estruturas dos diferentes modos de pensar, parecem ser abandonadas, conjuntamente com as reflexões sobre como os processos de representação e apresentação visual da 'informação' (dados) seriam aptos, ao mesmo tempo, para promover a percepção, a reflexão e o esclarecimento. $O$ foco no que foi produzido e não no como e quem o 'produz', o 'experimenta' e o 'interpreta', ou seja, no 'indivíduo' (ser), indica a crença ou a aposta em um 'sistema de informação' (objeto técnico), independente, desumanizado e estetizado, o qual só poderia existir em si mesmo, visto que as relações do 'indivíduo' (ser) com o 'sistema de informação' (objeto técnico), ou o outro, desaparecem na aparência das garbosas metáforas visuais de sucesso, que não deveriam ter um fim em si mesmas, mas apenas ser mais um meio de gerar autoreconhecimento para a contínua revelação e construção do próprio 'indivíduo' (ser).

Deste modo, a simples contemplação de qualquer 'sistema de informação' (objeto técnico) não poderia revelar nenhum dos processos inscritos no 'indivíduo' (ser), pois somente através da análise das relações existentes entre o 'indivíduo' (ser) e as 'informações' (dados) que poderia-se reconhecer o espaço de atuação das disciplinas de 'Arquitetura da Informação', do 'Design da Informação' e da 'Visualização de Informação'.

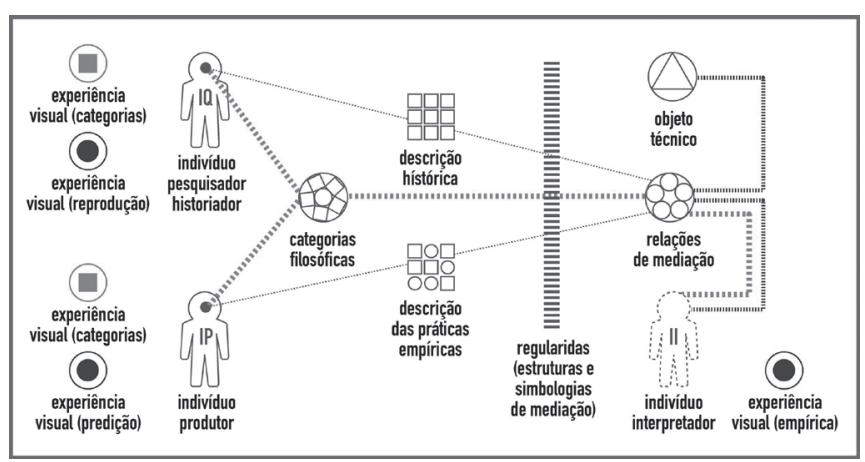

Figura 1: Modelo de observação e de inferência para análise dos 'sistemas de informação' (objetos técnicos). 
A inversão do ponto de observação, busca tentar entender o 'sistema de informação' (objeto técnico) a partir do 'indivíduo' (ser), para que se deixe de examinar o que se vê na superfície do 'sistema de informação' (objeto técnico), para que se passe a observar o que se induz, estimula ou produz no 'indivíduo' (ser), a fim de se identificar as regularidades e se descrever as nuâncias configuráveis das 'estruturas de mediação', que poderiam ser utilizadas do agenciamento dos processos de percepção aos processos de esclarecimento. Assim, o foco deixa de ser na análise da 'experiência visual' do 'indivíduo produtor' ou do 'indivíduo pesquisador', do que é representado e apresentado pelo 'objeto técnico', numa descrição histórica e/ou numa descrição das práticas empíricas dos resultados estéticos, para constituir-se como uma análise da 'experiência visual' do 'indivíduo interpretador', dos efeitos agenciados e sentidos durante as 'relações de mediação' com a composição e a organização do 'objeto técnico', presentes nos processos de percepção, de interpretação, de formulação do discurso e de proposição de ações, os quais poderiam ser observados e identificados no 'relato narrativo', conforme a figura 1.

\section{Metodologia}

Com o intuito de sustentar metodologicamente a abordagem, foi realizada uma revisão bibliográfica que contemplasse dois grupos distintos de autores: o dos teóricos da 'Arquitetura da Informação', do 'Design da Informação' e da 'Visualização de Informação', Andrea Resmini e Luca Rosati (2011), Johanna Drucker (2012) (2014a) (2014b), Manuel Lima (2011), Joel Katz (2012), Meg Durkerley (2013), Robert Jacobson (2000), Tamara Munzner (2015), Edward Rolf Tufte (2001a) (2001b) (2012), Isabel Meirelles (2013), Jesse James Garrett (2011), Katy Börner e David E. Polly (2014), Ary Moraes (2013), Julius Wiedemann (2012), Robert Spence (2007), Alberto Cairo (2013) e Nathan Yau (2011), que apresentam a história, os conceitos, os princípios, os modelos e as técnicas utilizadas para a concepção da representação, da apresentação e da visualização da 'informação' (dados), em desenvolvimento de 'sistemas de informação'; e o dos teóricos da fenomenologia, da percepção e da filosofia da técnica, Maurice MerleauPonty (2011), Bruno Latour (1986) e Gilbert Simondon (2007) (2010) (2012), que apresentam os conceitos e as proposições de 'redução fenomenológica', de 'indivíduo' (ser) no e em relação com o mundo (outro), de 'indivíduo transdutor', de 'estrutura de mediação' e de 'sistema cultural metaestável'.

Assim, o trabalho foi subdividido em 16 etapas de pesquisa: a análise e síntese das principais abordagens defendidas pelos autores; a análise e síntese das principais ideias e conceitos da abordagem fenomenológica; a análise e a descrição dos processos de assimilação da técnica; a análise e a descrição dos processos de percepção, interpretação e ação; a seleção do 'conjunto de informações' (dados), fatos históricos de um determinado momento histórico (Staatliches-Bauhaus) para a montagem dos 'sistemas de informação' (objetos técnicos); a organização e montagem do 'conjunto de informações' em 'sistemas de informação', na forma de 'classes dispersas', de 'infográfico' e de 'livro'; a organização dos 2 laboratórios para a realização das sessões de teste ('laboratório de exposição' e 'laboratório de relato'); a seleção de 15 participantes (alunos dos cursos de design), agrupados em 5 trios (5 alunos por 'sistema de informação'); a elaboração de uma 'ficha de observação'; a realização das sessões de teste no 'laboratório de exposição', no qual cada participante entra em contato com somente um 'sistema de informação' (1 sessão com até 45 minutos por participante, fotografada e com a realização de relatório de observação não participante); a realização das sessões de teste no 'laboratório de relato', no qual cada um dos participante desenvolve sozinho um 'relato narrativo' do que foi capaz de lembrar, de compreender e de narrar sobre as 'informações' do 'sistema de informação' (1 sessão com até 15 minutos por participante, filmada); a elaboração de uma 'ficha de interpretação'; a realização das sessões de debate sobre a experiência, com cada um dos trios (1 sessão de no máximo 20 minutos com 3 participantes, filmada); o preparo do 'quadro de frequência', com o resultado da frequência de uso das palavras; a análise, reflexão e discussão sobre os resultados; e finalmente, a descrição dos resultados obtidos no decorrer da pesquisa.

Denominou-se 'conjunto de informações' os textos primários, secundários e terciários, as imagens primárias, secundárias e terciárias e os elementos visuais e estruturais de organização espacial. Com o intuito de evitar que fatores como escala e proporção não influenciassem os resultados da pesquisa, as dimensões de todos os elementos textuais e visuais foram mantidas entre os três 'sistemas de informação'.

\section{Indivíduo (ser) e Informação (dados)}

O 'indivíduo' (ser) na condição de produtor (programador), de experimentador (usufruidor das experiências sensoriais) e de interpretador (ator propenso a ação) da 'informação' (dados), carregados de valores internos e externos ao 'sistema de informação', está sujeito a estados que caracterizam distintas fases da 'informação' (dados). Ou seja, como já apontava há algum tempo a 'ciência da informação' (Rengen, 2012, p.13), a 'informação' (dados) transmitida, transduzida ou traduzida tem o seu próprio estado transformado (Simondon, 2010), a 'informação' (dados) ganha diferentes valores durante o seu processamento, tanto para quem a articula como produtor, quanto para quem a articula como interpretador ou narrador. Então, as 'informações' (dados) começam a relevar as suas nuâncias, apresentam-se em condições que caracterizam os estados: de 'pré-informação', no qual todo o 'dado' está posto enquanto signo, sensação, sentimento e sentido percebido; de 'informação', no qual o 'dado' deixa de ser 'dado' para se tornar 'informação' destrinchada, conjugada e concatenada, enquanto conjunto de 'dados' processados e reprocessados, que instigam significados até ganharem o status de algum tipo de 'conhecimento' ou 'discernimento', na forma de uma rede aberta e ao mesmo tempo determinável de significados; e de 
'pós-informação', a "dita sabedoria" que leva a ação, derivada do domínio sobre as 'informações' discernidas, que induzem ou conduzem o 'indivíduo ao estabelecimento de habilidades e competência para a articulação da 'informação', que o levam até a propensão da ação.

Deste modo, o 'indivíduo produtor', das representações, das apresentações e das visualizações das 'informações' visuais, durante o encadeamento das 'informações', tem que propor e tentar montar processos de 'espacialização dos dados', que resgatem ou recuperem parte dos conhecimentos tácitos, das memórias culturais e dos processos fisiológicos, psíquicos e cognitivos, os quais poderão ou deverão ser executados pelo 'indivíduo interpretador', a fim de facilitar a sua compreensão e a sua geração de conhecimentos. Assim, aplica um conjunto de princípios e de técnicas advindas de três disciplinas: com a 'Arquitetura da Informação', busca estruturar, classificar e rotular ou etiquetar os 'dados', e acrescentar camadas extras de 'informação', necessárias aos processos de classificação, organização, armazenamento, resgate, seleção, conjunção, disjunção, conexão, sequenciamento, processamento e fluxo de 'espacialização dos dados' (Resmini, Rosati, 2011) (Lima, 2011) (Yau, 2011) (Tufte, 2001a) (Tufte, 2001b) (Tufte, 2012); com o 'Design da Informação', busca transformar todas as 'informações' em metáforas visuais análogas e/ou abstratas, que possam ser utilizadas para estruturar as representações, as apresentações e as visualizações da 'informação', traduzir um determinado conjunto de 'informações' num conjunto de elementos ou 'informações' visuais articuláveis, que possam depois de espacializados informar ou facilitar a geração de conhecimentos (Cairo, 2013) (Meirelles, 2013) (Wiedemann, 2012) (Dunkerley, (2013) (Jacobson, 200); e por fim, com a 'Visualização da Informação', busca modos e processos de assistência e de ensaio, da apresentação e da visualização da 'informação', que sejam capazes de estimular a geração de conhecimento e de sabedorias, capazes de promover a tomada de decisões e/ou de ações (Spence, 2007) (Cairo, 2013) (Garrett, 2011).

O 'indivíduo interpretador', diante das 'espacializações dos dados', representações, apresentações e visualizações da 'informação', concentra seus esforços na interpretação dos fenômenos, entre os estágios de 'concatenação' (o sentir e perceber os 'dados'), de 'crítica' (o processar criticamente a 'informação') e do impulso para a promoção da 'ação' (o atuar sobre o mundo).

Pode-se caracterizar o estágio da 'concatenação' como todo o processo de captação da 'informação' (dados), no qual são selecionados, conformados, conectados e/ou agregados a variantes ou condicionantes advindas: do meio, conjunto de circunstâncias momentâneas que constituem o ambiente; do corpo biológico, constituição específica de cada indivíduo; dos estados afetivos-emocionais, do 'indivíduo'; das variações no processamento fisiológico e cognitivo das 'informações'; dos hábitos pessoais e socioculturais inscritos no 'indivíduo'; das memórias resgatadas em cada instante; e das traduções entre distintos sistemas sígnicos (Merleau-Ponty, 2011). Visto que a percepção não pode ser caracterizada puramente como o instante ou o momento de captação, de um conjunto de informações, exatamente porque como processo complexo, está subordinada a um conjunto de níveis perceptivos, que devem ser integrados (Simondon, 2012).

Pode-se caracterizar o estágio da 'crítica' como o processo de reflexão, ponderação e síntese, no qual são realizadas, num processo de distanciamento do fenômeno, através de um conjunto de operações que permitem a 'construção' de uma ideia geral, estrutura tangível, ponto sólido, rede de relações entre 'informações' cristalizada momentaneamente, processo fruto de um certo tipo de julgamento ou seleção, que promove a redução e/ou a ampliação dos elementos originalmente analisados. A articulação do 'conjunto de informação' permite a consolidação momentânea de pareceres, que apagam as nuâncias, ou os excessos de 'informação', não úteis para a determinação de uma posição ou síntese.

Pode-se caracterizar o estágio da 'ação' como os processos de exteriorização ou exposição, no qual as ações partem do 'indivíduo' (ser) para o mundo (outro), a exposição condiciona o 'indivíduo (ser) e o mundo (outro) a transformação, por isso deixam-se ser capturadas, podem ser observadas, analisadas e ponderadas.

Assim, o 'relato narrativo' pode revelar os tipos de resultados que podem ser obtidos com determinados modos e processos de representação, apresentação e visualização dinâmica da 'informação'. A análise do 'relato narrativo' pode possibilitar a compreensão dos tipos de resultados que podem ser obtidos durante a exposição de um 'indivíduo interpretador' a um determinado 'sistema de informação', o domínio obtido sobre um determinado conteúdo, a capacidade de articulação e de compreensão dos significados, a capacidade de imaginar e promover significados, e a capacidade de revelar e promover 'estruturas de mediação', que anteriormente não tinham sido percebidas.

Logo, o 'indivíduo produtor' para cada projeto que desenvolve, deveria ser capaz de identificar os padrões 'estratégicos de obtenção da informação', característicos de cada 'indivíduo interpretador', a fim de ativar as reações e os efeitos, de curto e longo prazo, durante o processo de contato com a 'estrutura de mediação', que caracteriza cada 'sistema de informação'. Logo, se atribui a 'estratégia de obtenção da informação' em relação com a 'estrutura de mediação', o modo como cada 'indivíduo interpretador' pode interpelar, selecionar, organizar, conjugar, ler, interpretar e narrar as próprias experiências, conforme figura 02. 


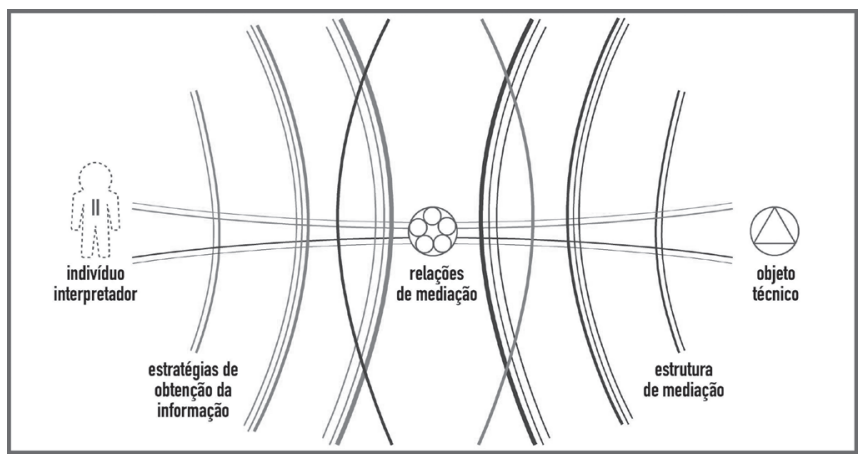

Figura 2: Relação 'indivíduo interpretador' e 'sistema de informação' (objeto técnico).

\section{Sistemas de Informação}

É importante lembrar que a separação artificial do 'conjunto de informações' não respeita o próprio caráter heterogêneo e dissipado da 'informação' (dados), no cotidiano do 'indivíduo' (ser), o que não assegura ou facilita uma situação ideal para as sessões de observação das 'relações de mediação', entre os 'indivíduos interpretadores' e os 'sistemas de informação' (objetos técnicos).

Contudo, os testes em laboratório foram realizados com três tipos de 'sistema de informação'. O 'sistema de informação' por 'classes dispersas', caracterizado como um 'conjunto de informações', classificadas e agrupadas, as quais precisam ser dispostas em função de uma 'concatenação' espacial que não foi totalmente definida previamente. O fluxo de obtenção da informação depende de como o 'indivíduo interpretador' estabelece um sistema inicial de referências, incrementa e descreve seus deslocamentos, seleciona, classifica, organiza, distribui e relaciona as 'informações', durante o processo de percepção, sequenciamento e planificação. Já, o 'sistema de informação' por 'infográfico', caracterizado como um 'conjunto de informações', classificadas, agrupadas e distribuídas, que foram previamente planificadas e espacializadas, na forma de sequências, grupos e subgrupos, que se relacionam e interrelacionam por disjunção, conjunção e/ou justaposição. E o fluxo de obtenção da informação depende, primeiro de como o 'indivíduo produtor' previamente definiu o sequenciamento e a distribuição na planificação das 'informações', e segundo de como o 'indivíduo interpretador' incrementa e descreve seus deslocamentos, percursos e saltos, que geram a seleção e a relação entre as 'informações'. Finalmente, o 'sistema de informação' por 'livro', caracterizado como um 'conjunto de informações', dispostas e 'concatenadas' previamente, que foram sequencialmente planificadas e espacializadas, e que devido a estrutura guiada pelo texto, precisam ser ativadas e sobrepostas sucessivamente. Assim, o fluxo de obtenção da informação depende, primeiro de como o 'indivíduo produtor' previamente definiu o sequenciamento e a sobreposição das 'informações', e segundo de como o 'indivíduo interpretador' incrementa e descreve seus deslocamentos guiado pela linha de texto e pelas sequencias de sobreposições, definidas pelo virar, abrir e

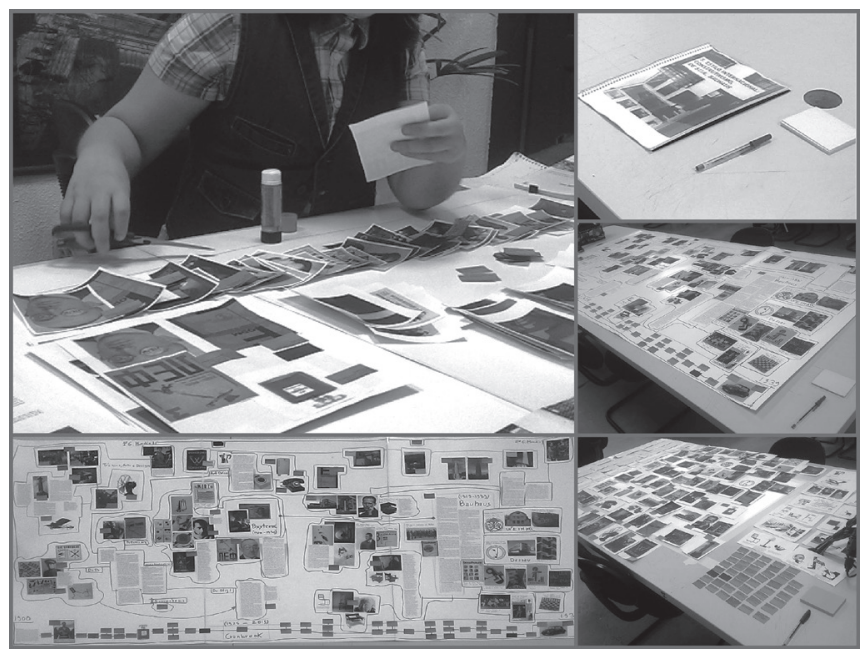

empilhar de páginas, conforme a figura 3.

Figura 3: Montagem do conjunto de informações (dados) em três 'sistemas de informação' ('classes dispersas', 'infográfico' e 'livro').

Os 'sistemas de informação' foram montados com o mesmo 'conjunto de informações' e propositalmente preparados para produzirem uma determinada sobrecarga de 'informação' no 'indivíduo interpretador', derivada da relação entre o número de 'informações' expostas em função do tempo prescrito.

\section{Testes}

Os testes foram realizados em três fases. Na primeira fase, foram realizadas as exposições dos participantes ao 'sistema de informação'. Na segunda fase, foram gravados os 'relatos narrativos' dos participantes. E na terceira fase, foram feitos debates em que os participantes relataram a percepção da experiência.

Durante o processo foram dadas as seguintes orientações: na introdução dos testes, "O objetivo do experimento é identificar os distintos comportamentos estimulados por cada tipo de 'sistema de informação'. Assim, tu serás primeiro exposto a um 'sistema de informação' e em seguida tu serás convidado a relatar o resultado da sua experiência"; na primeira fase, "Tu tens 45 minutos para observar, ler, estudar, anotar e organizar o 'sistema de informação' recebido", "Tu tens uma caneta e um bloco de notas para te ajudar a organizar toda a sua experiência com o 'sistema de informação'", "Após os 45 minutos, tu serás convidado a sair do laboratório para explicar o que foi compreendido sobre o 'sistema de informação', e "O tempo acabou. Por favor, venha comigo"; na segunda fase, "Tu tens até 15 minutos para relatar tudo o que conseguires lembrar ou compreender sobre o conteúdo que estava no seu 'sistema de informação", "Tente explicar do melhor modo as relações, as causas e as consequências existentes entre os fatos", "Tente ser o mais claro e objetivo que conseguires", "Tu consegues me explicar um modo de utilizar ou aplicar essas informações no seu dia-a-dia?", e, "Tu poderias me dar um exemplo prático para a utilização dessas 
informações?", e na terceira fase, finalmente, "Vocês podem me contar ou explicar quais foram as impressões que tiveram com os 'sistemas de informação'?", "Desculpem interromper, mas infelizmente o nosso debate acabou" e "Muito obrigado por participarem da pesquisa".

Durante a primeira fase foi utilizada uma 'ficha de observação', com a qual buscou-se descrever o comportamento recorrente observável dos participantes. E depois da segunda fase foi utilizada uma 'ficha de interpretação' dos 'relatos narrativos' com a qual tentou-se mapear: os fatos relatados existentes no material; os fatos relatados não existentes no material; os fatos relatados inventados ou falsos; as relações entre os fatos existentes no material; as relações entre os fatos não existentes no material; as relações entre os fatos inventados ou falsos; a construção de proposições entre 2 fatos ou entre 3 ou mais fatos (premissas); a construção de proposições por similaridade ou oposição; e a construção de proposições por meio da reprodução dos conteúdos presentes no 'sistema de informação' ou trazidas de outras fontes; e a construção de proposições inventadas ou falsas. Também foi montado um 'quadro de frequência', a partir da transcrição do 'conjunto de informações' conformadas pelos 'sistemas de informação' e a partir dos 'relatos narrativos', comparandose a frequência das palavras no 'sistema de informação' com a frequência do uso das palavras pelos participantes. E por fim, analisou-se em função de cada 'sistema de informação', as similaridades e as diferenças entre as narrativas.

\section{Resultados}

Os 'sistemas de informação' passaram por três processos de análise, caracterizados por três instrumentos: a 'ficha de observação', que ajudou a registrar o comportamento corporal e gestual dos participantes; a 'ficha de interpretação', que ajudou a destrinchar o 'relato narrativo' dos participantes; e o 'quadro de frequência', que ajudou a comparar a frequência do uso das palavras no 'sistema de informação' e no 'relato narrativo'.

Assim, ao comparar os 'sistemas de informação', em função dos registros do processo de observação do comportamento, pode-se argumentar que:

O'sistemadeinformação' por'classesdispersas' apresentou uma 'estrutura de mediação' excessivamente flexível, o que ao mesmo tempo, possibilita a articulação das classes de 'informação', mas deixava os participantes perdidos por não estabelecer nenhum tipo de discurso prévio. Os participantes sem saberem como ou por onde deviam começar a organizar, a captar e a interpretar o conteúdo, acabavam privilegiando, depois de algum tempo, a 'concatenação' por agrupamentos, em que os textos mantinham uma posição central, sendo rodeados pelas imagens. Toda a concentração mantida nas unidades de 'informação', contrastava com a dispersão do corpo, o qual intensamente rodeava e manipulava os blocos de 'informação'. Pode-se dizer, que o corpo demostrava precisar compensar a falta de uma 'estratégia de obtenção da informação', devendo tentar ajudar a montar uma estratégia motora rudimentar, que pudesse ser capaz de auxiliar o 'indivíduo interpretador', a adquirir alguma compreensão

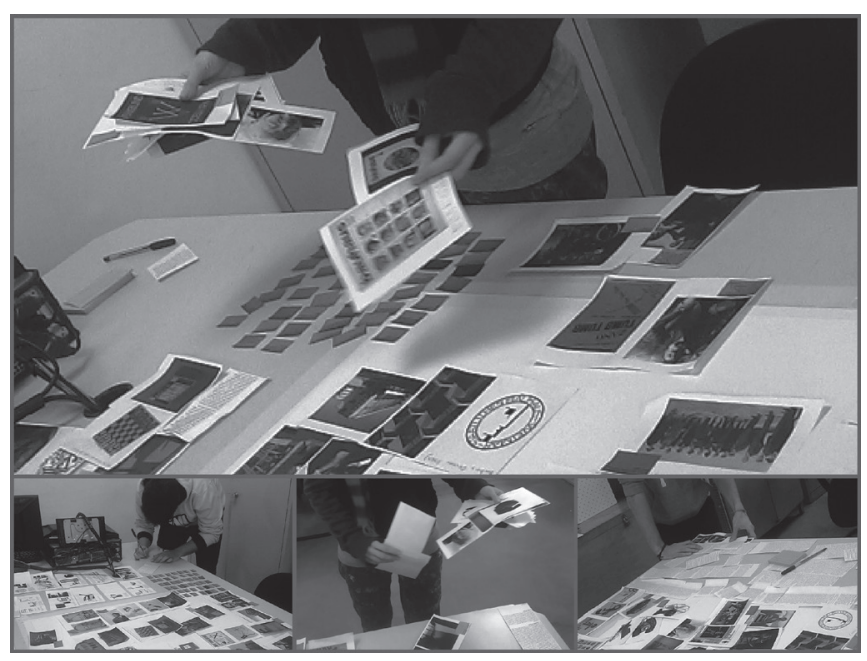

Figura 4: Teste de exposição ao 'sistema de informação' na forma de 'classes dispersas'.

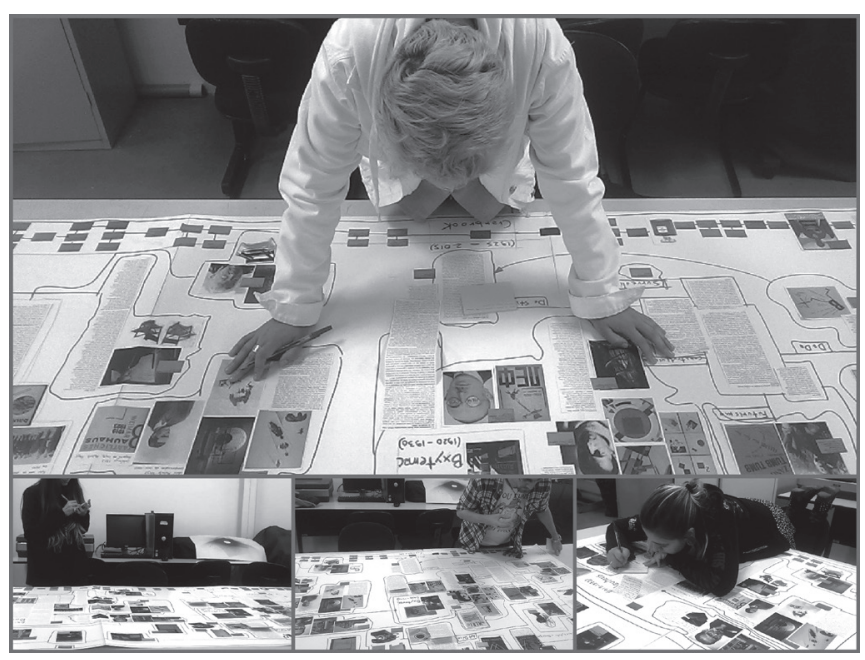

sobre o conteúdo, conforme figura 04.

Figura 5: Teste de exposição ao 'sistema de informação' na forma de 'infográfico'.

Já o 'sistema de informação' por 'infográfico' apresentou uma 'estrutura de mediação' parcialmente flexível, o que ao mesmo tempo, oferece e abre possibilidades de discurso e mantem algum tipo de fio condutor, que talvez seja formado pela definição da distribuição das classes de 'informação' em sequencias, agrupamentos e subdivisões familiares, as quais ajudam a organizar, a captar e a interpretar o conteúdo. 0 corpo participava de modo ativo do processo de interação com as unidades de 'informação', percorria o espaço planificado formado pelos blocos de 'informação'. Pode-se dizer que a 'estratégia de obtenção da informação' foi parcialmente estabelecida, e que com o passar do tempo, o 'indivíduo 
interpretador', tinha que várias vezes reestruturar a trajetória de observação para poder obter alguma compreensão sobre o conteúdo, conforme figura 05.

E finalmente, o 'sistema de informação' por 'livro' apresentou uma 'estrutura de mediação' socialmente disseminada, o que ao mesmo tempo, submete a uma estrutura lógica que facilita ao conduzir como um fio condutor e condiciona a sequência do encadeamento das classes de 'informação', que definem previamente um percurso que ajuda a organizar, a captar e a interpretar o conteúdo. $O$ corpo concentrado parece inerte ajudando o participante a manter uma maior concentração no 'sistema de informação'. Pode-se dizer que a 'estratégia de obtenção da informação' foi plenamente assimilada através do tempo pelo 'indivíduo

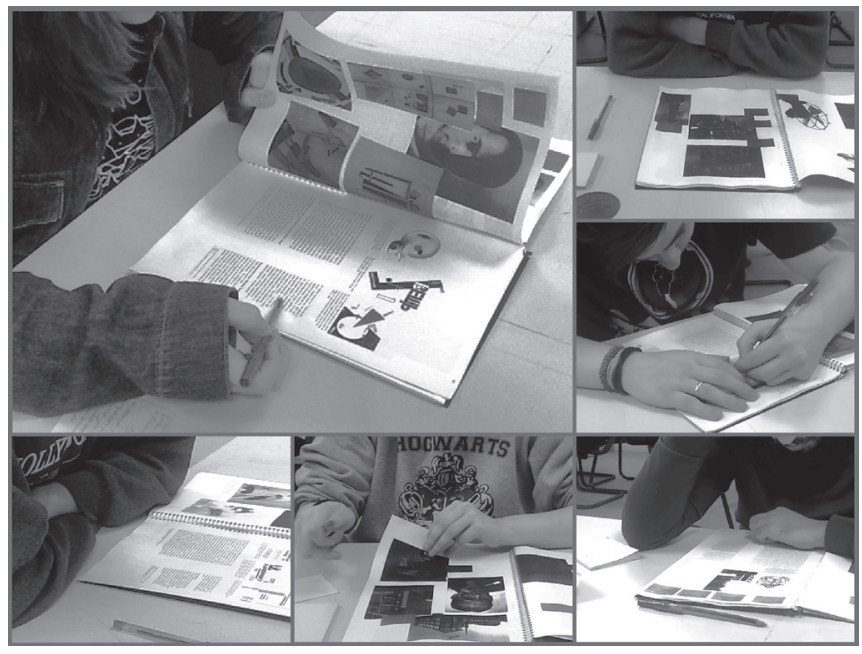

interpretador' que a regatava e a utilizava sem demostrar nenhum incomodo, conforme figura 06.

Figura 6: Teste de exposição ao 'sistema de informação' na forma de 'livro'.

Assim, ao comparar os 'sistemas de informação', em

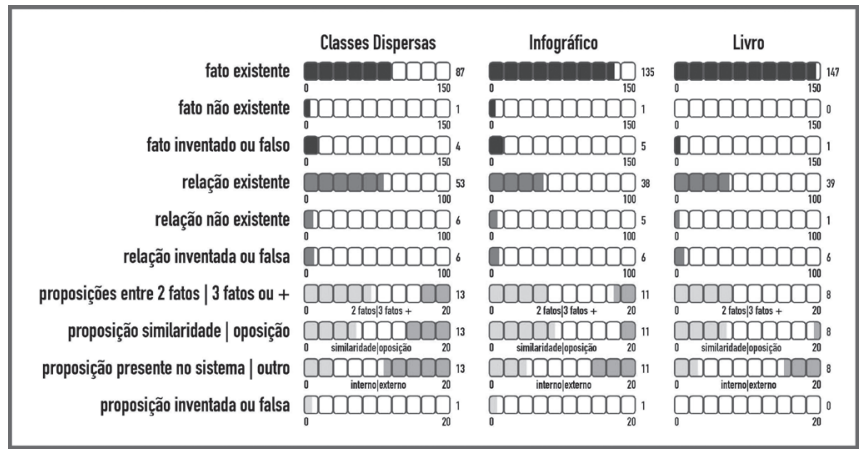

função do processo de interpretação do 'relato narrativo', pode-se argumentar, conforme a figura 7, que:

Figura 7: Análise dos dados dos 'relatos narrativos' sobre os 'sistemas de informação' ('classes dispersas', 'infográfico' e 'livro').

O 'sistema de informação' por 'classes dispersas' apresentou a maior inconstância nos 'relatos narrativos', com o seu foco dissipado em torno de pelo menos quatro pontos principais (arte e indústria | forma e função | movimentos artísticos | Bauhaus). Os 'relatos narrativos' destacaram a importância da formação de uma 'estratégia de obtenção da informação', que seja 'anterior' a 'execução do percurso' de observação, pois o foco tende a deslizar sobre as classes de 'informação' em trajetórias aleatórias ao primeiro instante. Mas o próprio 'relato narrativo' parece que ficou dependente de um tempo maior para sua formulação.

O'sistema de informação' por 'infográfico' apresentou com constância os 'relatos narrativos', com o seu foco em torno de dois pontos principais (movimentos artísticos | Bauhaus), mas com uma forte rede de conteúdos secundários (invenção do telegrama, lançamento do Chanel $n^{\circ} 5$, teoria da relatividade, primeiro plástico, entre outras informações que ilustravam o 'sistema de informação'). Os 'relatos narrativos' destacaram a importância da 'execução do percurso', o que evidência como estava parcialmente consolidada a 'estratégia de obtenção da informação', pois o foco era guiado pelos blocos principais de 'informação'. Mas, o 'relato narrativo' foi conduzido de modo fragmentado, com muita dificuldade em estabelecer relações ou conexões entre os 'fatos'.

O 'sistema de informação' por 'livro' apresentou a maior constância nos 'relatos narrativos', com o seu foco centrado em torno de dois pontos principais (relações sociais e políticas | movimentos artísticos e Bauhaus), sem variações relevantes na interpretação. Os 'relatos narrativos' puderam destacar a importância da sequência narrativa na definição da 'execução do percurso', o que evidência como já estava consolidada a 'estratégia de obtenção da informação', baseada na linha de texto e na sequência de páginas, pois foi possível o resgate de muitos 'fatos históricos existentes' e o foco proposto pelo 'indivíduo produtor', nas relações sociais e políticas que circunscreviam os movimentos artísticos que influenciaram a Bauhaus, conseguiu ser mantido, ou seja, reproduzido. Mas o 'relato narrativo' só foi capaz de reproduzir o discurso que existia no 'sistema de informação' sem conseguir estabelecer ao menos 'relações existentes' e promover 'proposições'.

Logo, ao comparar os 'sistemas de informação', em função da frequência de uso das palavras no 'sistema de informação' e nos 'relatos narrativos', pode-se argumentar que:

O 'quadro de frequência' de uso de palavra confirmou nos 'relatos narrativos' o uso e a reprodução das principais palavras presentes no 'sistema de informação'. Do mesmo modo, como apresentou a variação nos focos dos 'relatos narrativos'.

Deste modo, ao comparar os 'sistemas de informação', podem ser realizadas as seguintes colocações gerais:

- Os blocos de anotação foram sistematicamente acionados como um tipo de memória auxiliar dos participantes, durante a exposição aos três 'sistemas de informação'.

- Os textos foram privilegiados enquanto que as imagens foram sistematicamente descartadas, pois não chegaram a participar diretamente da construção dos 'relatos narrativos'.

- Ao mesmo tempo, que os 'sistemas de informação' parecem promover $\mathrm{o}$ aumento da capacidade de 
reproduzir 'fatos existentes', parece que perdem a capacidade de promover a conexão entre as 'informações', na forma de 'relações' e 'proposições'.

- As 'proposições' foram principalmente montadas a partir de similaridades ou semelhanças percebidas entre os 'fatos', sendo executadas poucas 'proposições' por oposição entre os 'fatos'.

- As 'proposições' promoveram principalmente relações entre dois 'fatos' e dificilmente entre 3 'fatos' ou mais.

- As 'proposições' apresentaram a tendência de não estarem condicionadas as proposições existentes nos 'sistemas de informação'.

- Durante os 'relatos narrativos' surgiram 'fatos' e 'relações' inventadas ou falsas, mas que não se pode estabelecer os motivos ou a sua relevância.

- Os participantes durante a obtenção da informação, parecem demostrar que o nível de dificuldade coincide com o nível de dispersão, e que o nível de facilidade coincide com o nível de concentração.

- Contudo, parece que o fator mais significativo constatado, seja que o acesso a cultura material, indicado pela diferença entre a renda dos participantes, anotada na 'ficha de observação', pesa mais do que todos os outros fatores e as próprias propriedades de cada 'sistema de informação'.

\section{Conclusões}

O estudo tentou contribuir com a identificação e descrição dos efeitos no 'relato narrativo' dos 'sistemas de informação', para o desenvolvimento futuro de ferramentas que possam ajudar a configurar as formas de representação, de apresentação e de visualização da 'informação'. Porém, ao mesmo tempo, tentou entender como manter ou promover um determinado grau de eficiência na transmissão da 'informação' e um grau de abertura que instigue um raciocínio dialético, a fim de fomentar processos de esclarecimento.

Os resultados parciais parecem indicar, apesar dos desvios contidos nas amostras, que as formas de representação, de espacialização e de visualização da 'informação', indicadas nas bibliografias consultadas, não revelam os efeitos que geram nos 'indivíduos interpretadores', pois esses modelos prescritos são contraditados pelos 'relatos narrativos', visto que somente caracterizam estilos de espacialização visual da 'informação', sem caracterizar sua eficácia ou explicar como podem ser assimilados pelos 'indivíduos interpretadores'.

Os 'relatos narrativos' demostram que infelizmente, a maioria dos participantes dos testes não estavam aptos a executar os três estágios de articulação da 'informação', 'concatenação', 'crítica' e propensão a 'ação', pois foram registradas raras 'proposições. Logo, parece que a reprodução do discurso do 'indivíduo produtor', tendo como contraponto a redução das 'proposições' pelo 'indivíduo interpretador', revela que o foco na replicação de 'fatos' limita a possibilidade ou propensão a 'ação'.

Contudo, a pesquisa torna claro, que as 'estruturas de mediação' devem considerar as diferentes 'estratégias de obtenção da informação', pois parece que a conformação social da 'estratégia de obtenção da informação' prevalece sobre as propriedades dos próprios 'sistemas de informação'.

A análise dos resultados revelou que por não terem sido realizadas várias sessões, com os mesmos participantes e os 'sistemas de informação', não teria-se como mensurar os efeitos acumulativos provocados pelos três 'sistemas de informação'. E deste modo, entender a influência que podem exercer as 'estruturas de mediação' dos 'sistemas de informação', quando aplicadas durante um período longo de tempo, a fim de verificar se as 'estruturas de mediação' poderiam conformar ou condicionar as 'estruturas de obtenção da informação' do 'indivíduo interpretador.

Assim, talvez o aprofundamento do estudo do 'relato narrativo' possa vir a produzir resultados que fundamentem a integração entre os processos perceptivos, fisiológicos, psíquicosecognitivoseas formas de produçãoda representação e apresentação e visualização da 'informação' (dados), de modo a legitimar os modelos dos 'sistemas de informação' (objetos técnicos) como instrumentos de esclarecimento. No entanto, este trabalho só foi capaz de apontar algumas nuâncias das 'estruturas de mediação' pelo 'relato narrativo'.

\section{Referências}

Börner, K.; Polley, D. E. (2014). Visual insights: a practical guide to marking sense of data. Cambridge, MA: The MIT Press.

Cairo, A. (2013). The functional art: an introduction to information graphics and visualization. Berkeley, CA: New Riders.

Carpendale, S. (2013). Innovations in visualization. In: Samavati, F. F., Hawkey, K. (Ed.). GI '13: Proceedings of the 2013 Graphics Interface Conference. Toronto, TO: Canadian Information Processing Society Toronto. Retrieved from: http://innovis.cpsc.ucalgary.ca/innovis/uploads/ Publications/Publications/13-Carpendale-GI.pdf.

Carusi, A. (2010). Technologies of representations: images, visualizations and texts. In: Proceedings of the Computational turn. Enschede, EN: University Twente. Retrieved from: http://athanasius.stanford.edu/Readings/ Carusi.pdf.

Drucker, J. (2012). Humanities approaches to graphical display. In: Swanstrom, L., Pressman, J. (Ed.). Digital Humanities Quarterly - DHQ. Providence: Alliance of Digital Humanities Organizations, 2011. Retrieved from: http://www. digitalhumanities.org/dhq/vol/5/1/000091/ 000091.html.

Drucker, J. (2014a). Graphesis: visual forms of knowledge production. Cambridge, MA: Harvard University Press.

Drucker, J. (2014b). Visualizing Interpretation. Los Angeles: Department of Information Studies. Retrieved from: http:// polaris.gseis.ucla.edu/drucker/VisualizingInterp _07.pdf.

Dunkerley, M. (Ed.). (2013). Information visualization: perception for design. Waltham, MA: Morgan Kaufmann Publishers.

Garrett, J. J. (2011). The elements of user experience: usercentered design for the web and beyond. Berkeley, CA: New Riders Publishing. 
Jacobson, R. (Ed.). (2000). Information design. Cambridge, MA: The MIT Press.

Katz, J. (2012). Designing Information: human factors and common sense in information design. Hoboken, NJ: John Wiley \& Sons, 2012.

Latour, B. (1986). Visualization and Cognition: drawing things together. In: Kuklick, H. (Ed.) Knowledge and Society Studies in the Sociology of Culture Past and Present. Greenwich, GR: Jai Press, Vol. 6, 1986, pg.01-40. Retrieved from: http://www. bruno-latour.fr/sites/default/files/21-DRAWING-THINGSTOGETHER-GB.pdf, http://hci.ucsd.edu/10/readings/ Latour(1986).pdf.

Lima, M. (2011). Visual complexity: mapping patterns of information. New York, NY: Princeton Architectural Press.

Meirelles, I. (2013). Design for information: an introduction to the histories, theories, and best practices behind effective information visualizations. Beverly, CA: Rockport Publishers. Merleau-Ponty, M. (2011). Fenomenologia da Percepção. São Paulo, SP: Editora WMF Martins Fontes.

Moraes, A. (2013). Infografia: história e projeto. São Paulo, SP: Blucher.

Munzner, T. (2015). Visualization analysis \& design. Boca Raton, FL: CRC Press. Murray, S. (2013). Interactive data visualization for web. Sebastopol, CA: O’Reilly Media.

Rengen, S. Introdution. In: Wiedemann, J. (Ed.). (2012). Information graphics. Köln, RNV: Taschen GmbH.

Resmini, A., Rosati, L. (2011). Pervasive information architecture: designing cross-channel user experiences. Burlington, MA: Morgan Kaufmann.

Rosenberg, D., Grafton, A. (2010). Cartographies of Time: a history of the timeline. New York, NY: Princeton Architectural Press.

Simondon, G. (2007). El modo de existencia de los objetos técnicos. Buenos Aires, BA: Prometeo Libros.
Simondon, G. (2010). Communication et information: cours et conférences. Paris, RP: Les Éditions de la Transparence.

Simondon, G. (2012). Curso sobre la percepción. Buenos Aires, BA: Editorial Cactus.

Spence, R. (2007). Information Visualization: design for interaction. Harlow, HW: Pearson Prentice Hall.

Tufte, E. R. (2001a). Envisioning information. Cheshire, CT: Graphics Press LLC.

Tufte, E. R. (2001b). Visual display of quantitative information. Cheshire, CT: Graphics Press LLC.

Tufte, E. R. (2012). Visual Explanations: images and quantities, evidence and narrative. Cheshire, CT: Graphics Press LLC, 2012.

Wiedemann, J. (Ed.). (2012). Information graphics. Köln, RNV: Taschen $\mathrm{GmbH}$.

Yau, N. (2011). Visualize this: the flowingdata guide to design, visualization, and statistics. Indianapolis, IN: Wiley Publishing.

Zuk, T; Carpendale, S. (2006). Theoretical analysis of uncertainty visualizations. In: Proc. SPIE \& IS\&T Conference electronic imaging, Vol. 6060: Visualization and Data Analysis 2006. New York, NY: AIP Publishing LLC. Retrieved from: http://innovis. cpsc.ucalgary.ca/innovis/uploads/ Publications/Publications/Zuk_2006_TheoreticalAnalysis. pdf.

Zuk, T., Schlesier, L., Neumann, P., Hancock, M. S., Carpendale, S. (2006). Heuristics for information visualization evaluation. In: BELIV 2006: Proceedings of the workshop beyond time and errors: Novel evaluation methods for information visualization, held in conjunction with the working conference on advanced visual interfaces (AVI 2006). Venice, VE: ACM Press. Retrieved from: http://innovis.cpsc.ucalgary. ca/innovis/uploads/ Publications/Publications/Zuk_2006_ HFI.pdf. 